\title{
Static Data Structures
}

\section{Reconciling Template Metaprogramming and Generic Programming}

\author{
Michael C. Burton \\ Open Harbor \\ 1123 Industrial Rd. \\ San Carlos, CA 94070 USA \\ mburton@openharbor.com
}

\author{
William G. Griswold \\ Department of Computer Science $\& 6$ Engineering \\ University of California, San Diego \\ La Jolla, CA 92093-0114 USA \\ wgg@cs.ucsd.edu
}

\author{
Andrew D. McCulloch and Gary A. Huber \\ Department of Bioengineering \\ University of California, San Diego \\ La Jolla, CA 92093-0412 USA \\ \{amcculloch, ghuber\}@ucsd.edu
}

\begin{abstract}
C}++$ is decidedly asymmetric in its approach to the static and dynamic aspects of the language. As a consequence, techniques that we develop using one aspect (e.g., for loops with STL iterators) will not work with the other (Template Metaprogramming for the static side of $\mathrm{C}++$ ) due to syntax differences in the way each is defined and used [11]. These incompatibilities complicate switching between dynamic and static implementations of a behavior. Thus, for example, it is difficult to optimize a bottleneck by making a straightforward dynamic implementation static.

This paper introduces Static Data Structures, a template metaprogramming implementation of arrays that permits easily swapping in and out generic programming container implementations, giving a programmer precise control over the tradeoff between run-time costs and
\end{abstract}

The original version of this chapter was revised: The copyright line was incorrect. This has been corrected. The Erratum to this chapter is available at DOI: 10.1007/978-0-387-35672-3_13 
compile-time costs throughout the phases of software development. A mechanism to interface Static Data Structures with the generic programming algorithms of the STL is provided. This technique was used in the implementation of a highly flexible finite element method framework, resulting in two orders of magnitude speed-up in performance via substitution.

Keywords: template metaprogramming, generic programming, C++, STL, Boost, partial evaluation, object-oriented numerics, static data structures, heterogenous type lists, finite element method, FEM

\section{Introduction}

Generic Programming (GP) has opened doors in the $\mathrm{C}++$ scientific computing community by allowing developers to standardize on wellunderstood modules and algorithms that also perform well. Many GP techniques have been adapted from the Standard C++ Library and the works of other GP authors to create systems that execute quickly, but are also easy to extend (arguably a primary goal of modularity).

We have used many of these techniques in our own software, the Finite Element package Draco. The Finite Element Method (FEM) is a method of scientific computation for solving complex equations through numerical means, populating matrices of data using equations that represent the problem to be solved and then solving the resulting matrices. It is traditionally implemented using multi-dimensional containers (e.g., arrays) populated by either function pointers or hard-coded functions. By using functors, traits, iterators, and other GP techniques, we've significantly modernized Draco versus its Fortran predecessor with the end goal of increasing performance and decreasing the cost of extension.

But GP could not achieve all the performance gains that we hoped. Draco is targeted for use in bioengineering research, which requires an unusual amount of adaptability to a wide range of "structures" modeling problems: rigid, flexible, electrical, chemical, etc. The motivation for our research began with the need to implement a high-performance Finite Element Method software package that was also easy to customize for different applications. To get the desired flexibility, we wished to use GP techniques for representing arrays of functions. However, this resulted in function-pointer references, which compilers were not able to inline. Because these functions appeared in the innermost loops of the solution method, and these functions were quite simple (often returning a constant or a simple linear form such as $1-\mathrm{x}$ ), this unacceptably compromised performance.

Consequently, we resorted to the emerging techniques of Template Metaprogramming (TMP) to get the highest possible performance out 
of our innermost loops. More specifically, we used TMP to produce functions and classes templatized on parameters that would traditionally be run-time variables. This allowed us to vastly improve performance of these objects by specifying key variables at compile time.

However, there's a wide gap between the patterns used by GP and those used by TMP, due to the dissimilarity of the syntaxes used to invoke them. Code that is highly optimized by using Template Metaprogramming typically cannot take full advantage of the abstractions developed in GP, and vice versa. For example, we needed ways to do common GP tasks such as iterate over a range of TMP objects, which is impossible with existing GP technology since the value being iterated over is a compile-time value.

Without compatibility to standard GP interfaces such as the STL, it was not possible to easily switch between TMP and GP implementations of the same behavior. This meant making early decisions about what parts of the system should use TMP and which should use GP. Early use of TMP complicates development and pushes up compile times, slowing development. Late use of GP harms run-time performance. Ideally, we would like to build a system using GP, run performance tests, and then optimize by a straightforward substitution of GP implementations for TMP ones at strategic points.

In this paper we demonstrate a method to reconcile GP and TMP and solve this specific problem by providing a Template Metaprogramming mechanism standardized on the STL interface. The advantage of this approach is that for little effort, highly optimized Template Metaprogramming containers and algorithms can be swapped in for standard STL to increase performance. In order to develop this technique, we needed to invent a new kind of container, which we call a Static Data Structure, that could contain objects of different types. Section 6 reviews the technique with a focus on introducing it into existing code. We present our empirical performance measurements in Section 7, followed by a complete example in Section 8 . We close with discussion and our conclusions in Sections 9 and 10.

\section{Related Work}

Czarnecki and Eisenecker introduced heterogenous type lists [3] to store references to objects of unique types in a recursive list-like data structure. Eisenecker extended these lists to support values in his presentation of heterogenous value lists [5].

Burton also extended these type data structures to support values, and developed algorithms that are identical in call syntax to those in 
the STL [2]. His implementation of Static Data Structures uses arraylike template definitions rather than the the recursive list-like structures of Eisenecker.

Winch [13] similarly developed STL-compatible algorithmic access to value lists called VarLists, which she then employed to generate and access $\mathrm{C}++$ class definitions. Gurtovoy has developed algorithms that work with type lists, and has extended the implementation to support a variety of algorithms in the MPL [6], a library included as part of the Boost libraries [1].

This paper asserts the critical advantage of using these techniques to swap in improved implementations of modules later in the development cycle when they are less costly to compile. Its contribution is to provide a case study and performance evaluations for a real software system that highlight the benefits of using this technique in practice.

\section{Template Metaprogramming}

Template Metaprogramming (TMP) is a technique that uses $\mathrm{C}++$ templates to perform computations at compile time that would ordinarily be performed at run-time. It is an example of partial evaluation, a method of transforming one program into another by restricting its input domain and generating a more specialized program that can run faster [11]. Jones, et al. assert the utility of partial evaluation to optimize scientific computation [8]. TMP is the most accessible form of user-controlled partial evaluation available to $\mathrm{C}++$ programmers, as its mechanisms are a part of the standard language.

A simple example of TMP is the following class, which computes $x^{N}$ at compile-time for integer values of $x$ and $N$.

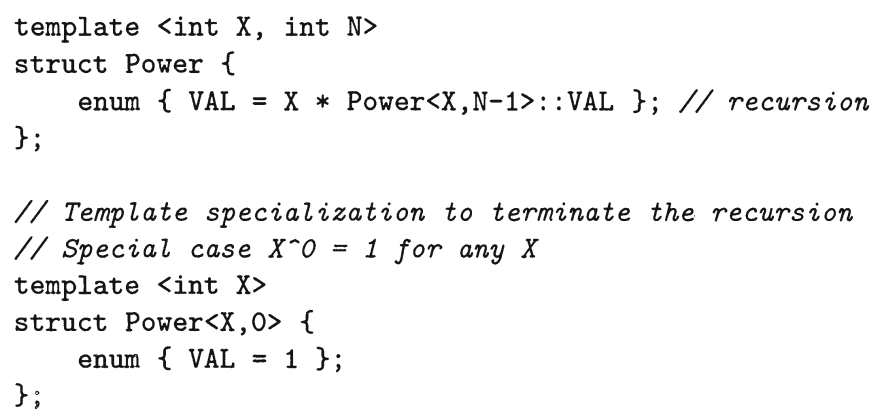

Listing 1: Template Metaprogramming: Power example

To use the code, one would simply get the value of the enumerated value Power $\langle\mathrm{X}, \mathrm{N}\rangle:$ : VAL substituting appropriate values of $\mathrm{X}$ and $\mathrm{N}$. A more 
comprehensive example of TMP may be found in Todd Veldhuizen's Expression Templates package [12].

TMP is a powerful tool for improving the performance of programs used for purposes of scientific computation (or anywhere that speed is highly desirable). However, its syntax is sufficiently different from the syntax of traditional $\mathrm{C}++$ programming that introducing it into an existing program can often be of questionable benefit when compared against the cost of implementation.

The problem is as follows: in template metaprogramming it is common to parameterize a function, class, or functor, on one or more integer parameters (e.g., the Power $<\mathrm{X}, \mathrm{N}\rangle$ example above). The performance benefits of parameterization can be profound for operations that are nested within multiple layers of loops, as is common in scientific programming. This improvement in run time performance can come at the cost of a significant increase in compilation time, as other TMP library designers have noted [7]. We will address this tradeoff later in Section 7.

Now suppose we wish to compute the first $n$ values of $x^{n}$ and store them for later use. In a traditional $\mathrm{C}++$ program, we would most likely create a data structure to hold the values and then loop over the standard $\mathrm{C}$ function call to compute pow $(\mathrm{x}, \mathrm{n})$ for each position in the container, like so:

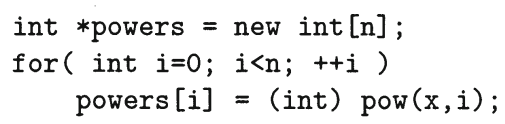

However, it is impossible for us to iterate like this with our TMP Power $<\mathrm{X}, \mathrm{N}>$ function, since $\mathrm{N}$ in this case is a compile-time parameter and cannot be varied at run-time.

Traditionally, we have two choices. The first is to manually list out each call to the Power $<\mathrm{X}, \mathrm{N}>$ function in our source code, effectively unrolling the loop manually ourselves. This is neither scalable nor easy to maintain.

The other choice is to use template metaprogramming to construct a recursive loop (the same technique used in the Power $\langle\mathrm{X}, \mathrm{N}\rangle$ example above) that is evaluated at compile time and used to populate the data structure. This is the typical solution when using TMP, as it tends to be consistent with other TMP solutions extant within a system. However, its syntax is significantly different from that of the original $\mathrm{C}++$ solution, and so it is difficult to introduce into an existing non-TMP system. Really all we want to do is lay out our TMP functions in some sort of sequence that we can iterate over, but this presents complications, as discussed in the next section. 


\section{Static Data Structures}

One approach to creating an iteratable data structure might be to construct something like an array of function pointers, whereby each item in the array contains a pointer to one of our Power $<\mathrm{X}, \mathrm{N}>$ functions. However, because the template instance of Power $<\mathrm{X}, \mathrm{N}\rangle$ for each value of $\mathrm{N}$ is actually a distinct type, we can't make a single array to hold more than one function. If we resort to inheritance tricks to enforce a common superclass type with indirections to virtual methods, we lose the performance benefits of TMP.

We have made some progress in joining high performance with modularity by means of Generic Programming, by introducing the concept of Static Data Structures (SDS). A static data structure is conceptually similar to a typical data structure, but it uses templates to organize its elements at compile-time rather than at run-time, thus incurring little if any run-time overhead. In addition, the elements of a static data structure may be types rather than just objects.

Later we will show how to use static data structures with generic programming algorithms such as those found in the STL. This will allow us to develop our code using standard STL algorithms, but subsequently introduce static data structures into critical portions of the code at low cost.

Let's begin our introduction to static data structures with an example of a regular data structure instead. Here we present a standard C array containing three elements:

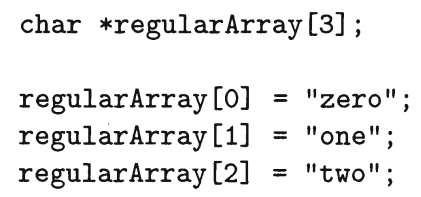

The array consists of three contiguous locations in memory, each containing the address of a static string. Very similarly, our static data structure will resemble an array like the one above. It will also contain three elements (although there's no guarantee that they will be held contiguously in memory):

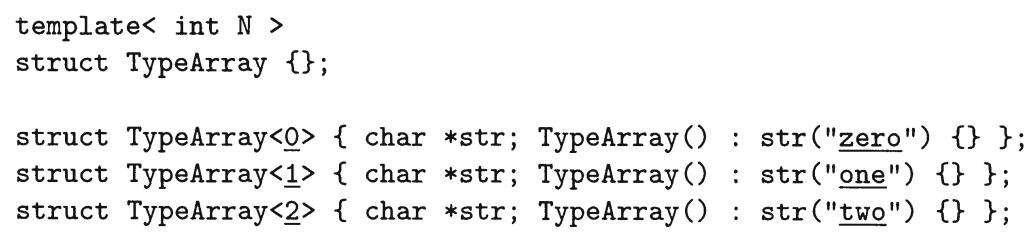

Listing 2: First Implementation of a TypeArray 
In the TypeArray example above, every item in the TypeArray has a variable called str. Any instance of TypeArray<0> will have str point to the "zero" string. Likewise, any instance of TypeArray<1> will be "one" and any instance of TypeArray<2> "two". This allows the programmer to index into the TypeArray much as if it were an actual array. The usage of each is fairly similar. The following code snippet will print out 'one' twice:

cout $\ll$ regularArray [1] $\quad<$ endl;

cout $\ll$ TypeArray<1>().str $\ll$ endl; // Instantiate a TypeArray

// element and access its str

The benefit of TypeArray over traditional arrays is that it can store multiple elements, each of a unique type. But before we address that question in full, we will first need to fix one flaw with the TypeArray class as it stands.

TypeArray as it is shown above is basically a pattern rather than an actual implementation. This is because the TypeArray declaration can only be used once inside a program module due to the global scope of the TypeArray type declaration.

The solution to this problem is to use a "Tag" type to partition the TypeArray space. Multiple TypeArrays may now exist in a single program, each identified using a unique tag. The Tag is simply any data type that is used as a template parameter to identify a single TypeArray. Here is the modified TypeArray class and two example usages:

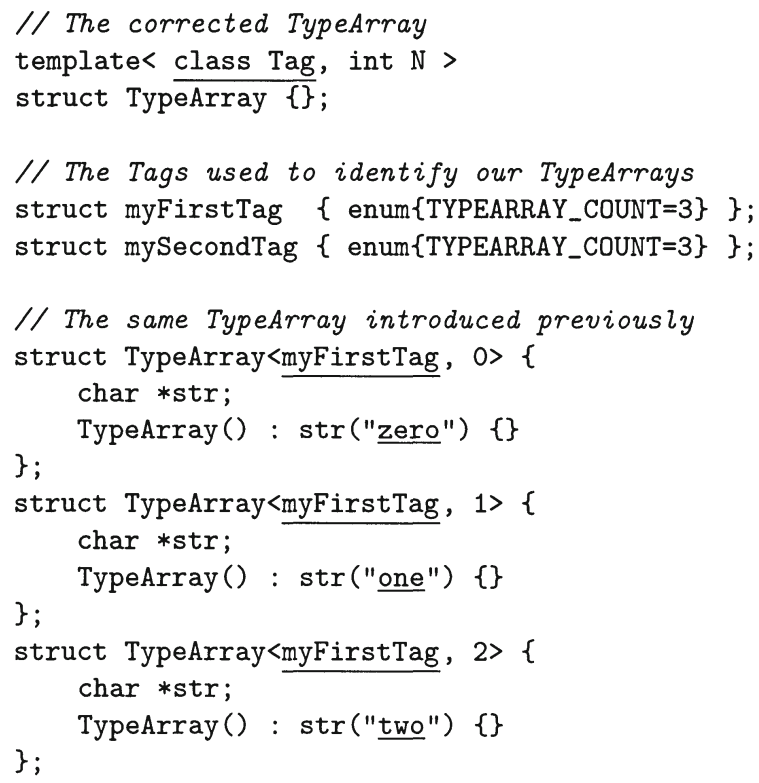

Listing 3: Tagged TypeArray, First Example Array 
// A second TypeArray, this one using types instead of objects struct TypeArray<mySecondTag, $0>\{$ typedef int item_type; $\}$; struct TypeArray<mySecondTag, $1>\{$ typedef double item_type; $\}$; struct TypeArray<mySecondTag, $2>\{$ typedef char item_type; $\}$;

Listing 4: Tagged TypeArray, Second Example Array

The Tag serves another purpose in addition to identifying the TypeArray. It also indicates how many elements are in the TypeArray. We can't store this number in the TypeArray itself because there is no single class that represents the entire TypeArray (since TypeArray is a family of classes that represent one data structure). TYPEARRAY_COUNT is an enumeration type instead of an integer because an enumeration is guaranteed to be immutable and known at compile-time (and therefore valid as a template parameter). Later we'll introduce algorithms that use the TYPEARRAY_COUNT in the Tag to determine the proper range of elements to iterate over.

Using this tag technique, TypeArray is ready to be used in real programs. The reader may notice that we've introduced a new technique in Listing 4. In addition to storing objects, TypeArrays can also store type information. This is what makes TypeArrays different from regular data structures.

To realize the power of this technique, we'll need to introduce a convention into the TypeArray definition by adding an object of type item_type to each element in the TypeArray:

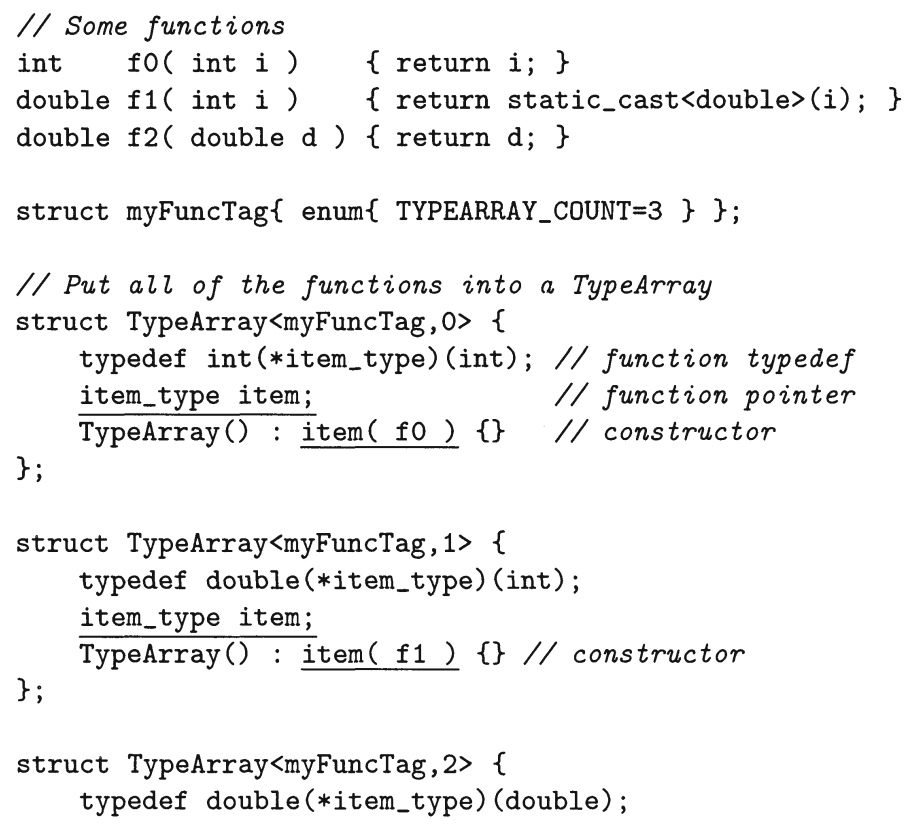




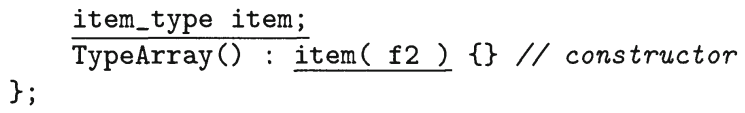

Listing 5: TypeArray storing objects of different types

This extension removes the responsibility of instantiating objects of type item_type away from the module using the TypeArray. Without it, a module that wanted to iterate over the items in a TypeArray would have to additionally know how to instantiate those items, an unreasonable expectation for most modules.

An example of a function that might use this technique could be:

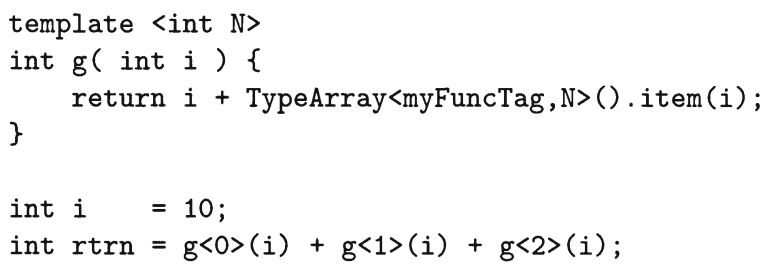

Listing 6: Example of a function using a function from a TypeArray

$\mathrm{g}()$ is parameterized on an integer value of $\mathrm{N}$, selecting the function to invoke. The programmer who implemented this function won't necessarily know the return type of the function TypeArray<myFuncTag, $N>()$. item () (which corresponds to the return type of either fo(), f1(), or f2()), but her $\mathrm{g}()$ function will work correctly regardless. We can then iterate over the values of $\mathrm{N}$ to sum up the computation of $\mathrm{g}()$, even though the types of the underlying functions are all different ${ }^{1}$.

With this last modification to TypeArray, we now have a complete system for storing "arrays" of objects, each of which can be its own unique type. This gives us a sort of compile-time polymorphism where each object in the structure can have its own unique type signature but still conform to the same concept. This will be important to us in the next section; there we will need to iterate over collections of uniquelytyped functors.

The complete source code for TypeArray may be found in the appendix of Section 10.

We still need to show how to iterate over these static data structures, at which point they will become significantly more useful. When that

\footnotetext{
${ }^{1}$ This example assumes that there exists an appropriate type conversion between all of the return types of the various $\mathrm{g}($ ) functions, since they may be arbitrarily different.
} 
happens we'll be able to take full advantage of TypeArray structures that behave like arrays of objects but are capable of doing significantly more. Until then, we are limited to using contrived examples to demonstrate their usefulness.

\section{Generic Programming with Static Data Structures}

The goal of this section is to introduce algorithms that can operate on TypeArrays, just like the STL has algorithms that can operate on STL containers. Additionally, we want to ensure that the interfaces we use for our algorithms are compatible with those of the STL, enabling us to transparently substitute static data structures for STL containers when using these algorithms.

Ideally, we would like to create versions of our static data structures that could interoperate with the standard STL algorithms, thereby allowing us to immediately take advantage of the full range of algorithms already in existence.

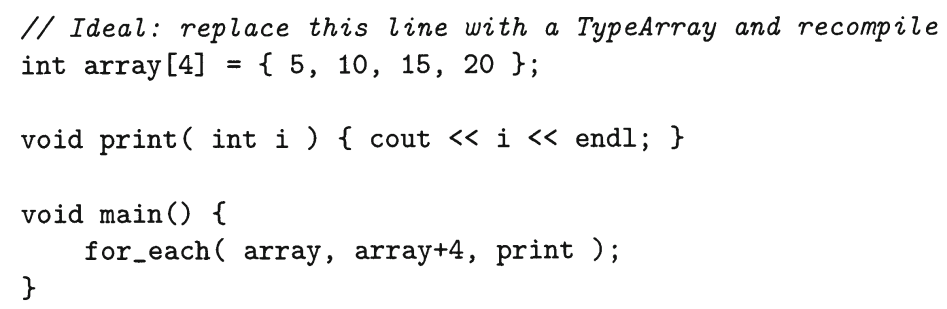

Unfortunately, this is impossible. Whereas the same operator is applied over elements of uniform type in a GP STL algorithm, our TypeArrays by design apply different operators to different elements. The STL iterator interfaces make no provision for specifying these operators.

Instead, we've created several static data structure algorithm interfaces, each of which is interface-compatible with an STL counterpart. This means that any module that uses these algorithms doesn't necessarily need to know whether the structures it's operating on are STL containers or static data structures. Using this technique, a user can swap static data structures with STL containers when they call a given algorithm and never need to know how the algorithm is doing its magic.

Let's begin with the for_each() algorithm from the STL. Just as one would use for_each() on an STL container, the usage of for_each() on TypeArrays is similar:

// Reference TypeArray<myFuncTag,N> definitions

// from previous section

TypeArray<myFuncTag, $0>$ tarray; // TypeArray object instantiation 
struct myOperatorTag\{\} // Tag for the algorithm

for_each( begin(tarray), end(tarray), myOperatorTag());

Listing 7: Example for_each() usage

The myOperatorTag type is another tag, but instead of tagging the TypeArray, myOperatorTag identifies the sequence of operators that for_each() will use. This enables the for_each() algorithm to index into a sequence of operators, one per element in the TypeArray. This is necessary because every element in the TypeArray may be of a unique type, and so there may need to be a unique operator for each.

Otherwise, the syntax is fairly similar. We call begin() on a TypeArray, as well as end(), to mark the range of elements that for_each() will be operating on (the range is obtained from the myFuncTag's TYPEARRAY_COUNT value). We pass the algorithm a pointer to a set of operators, and expect those operators to be executed on each element in the TypeArray.

Here is the for_each() generic algorithm for TypeArrays. The full implementation may be found in the appendix, Section 10.

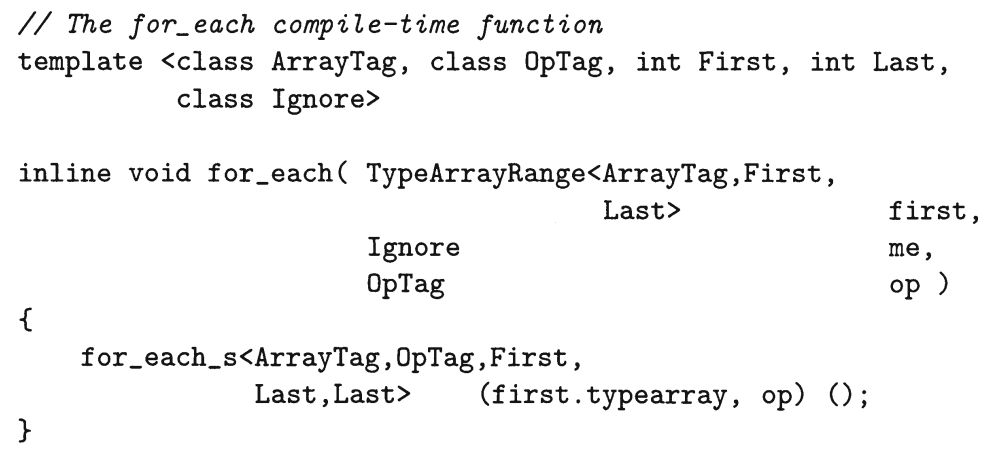

Listing 8: for_each() algorithm implementation

The implementation for for_each() and its related structures will get fairly dense, so we'll proceed by describing each of the above source code elements in turn.

ArrayTag. A Tag that indexes into the TypeArray of elements to be iterated over. It uniquely identifies the TypeArray that the user wishes to operate on.

OpTag. A Tag that indexes into a sequence of operators, each of which will be invoked on one of the items in the TypeArray of elements. 
The OpTag is used by for_each_traits to identify the sequence of operators. We'll discuss for_each_traits shortly.

TypeArrayRange. A class that indicates the range of the TypeArray over which the for_each() algorithm is to iterate. The non-member function begin() takes a TypeArray and returns a TypeArrayRange by asking the TypeArray's Tag. The code for TypeArrayRange may be found in Appendix 10.

First, Last. Integer parameters that indicate the range of elements to be iterated over. First and Last are automatically inferred by the compiler from the values of First and Last in the TypeArrayRange.

Ignore. This parameter is always ignored. Our implementation of for_each() ignores the second iterator argument, and it is only included for reasons of interface compatibility with the STL.

for_each_s. A helper class that recursively calls itself Last - First times, invoking an operator from the list of opTag operators for each element in the TypeArray.

The for_each() function itself is relatively simple. The actual work done by the algorithm is implemented in the for_each_s support structure. Here is its implementation: 
// Recursive for_each support structure, for_each_s template <class ArrayTag, class OpTag, int First, int Last, int ReverseIndex >

struct for_each_s<ArrayTag, OpTag, First, Last, ReverseIndex $>\{$ enum $\{$ Index $=$ (Last - ReverseIndex) $\}$;

// Constructor

for_each_s ( TypeArray<ArrayTag,First> \&first, OpTag \&optag ) : _first(first), _optag (optag) \{\}

void operator()() \{

// 'look up' the operator class in for_each_traits and // do the operation on the item for_each_traits<0pTag, Index $>$ (_optag).op( ) TypeArray<ArrayTag,First+Index> () . item

// Instantiate the next for_each_s in the sequences for_each_s<ArrayTag, OpTag, First, Last, ReverseIndex-1> next(_first,_optag);

next (); // Here is the recursion \}

protected:

TypeArray<ArrayTag,First> \&_first;

\} ;

OpTag \&_optag;

// Terminate the recursion for for_each_s when ReverseIndex=0 template <class ArrayTag, class OpTag, int First, int Last, int ReverseIndex >

struct for_each_s<ArrayTag, OpTag, First, Last, $\underline{0}>\{$

enum $\{$ Index $=$ Last - ReverseIndex $\}$;

for_each_s ( TypeArray<ArrayTag,First> \&first, OpTag \&optag ) : _first(first), _optag(optag) \{\}

void operator()() \{

I/ do the operation on the item

for_each_traits<0pTag, Index> (_optag).op (

TypeArray<ArrayTag, First+Index>() . item ) ;

// Terminate. Do not recurse further \}

protected: 
TypeArray<ArrayTag,First> \&_first;

\};

OpTag \&_optag;

Listing 9: for_each_s implementation

The for_each_s class is complex, but its goal is simple, to execute an operator for every element in the TypeArray. It does so by recursively instantiating itself and executing an operator (from a sequence of operators) for each element in the TypeArray. To do so, it makes use of the following objects and parameters:

ReverseIndex. An index that begins at Last - First and decreases down to 0 with every level of recursion. It is the complement of Index. ReverseIndex is used by for_each_s to know when to terminate the recursion.

Index. An index that begins at 0 and is increased with every instantiation of for_each_s. Index is a convenience value computed from ReverseIndex.

TypeArray $<\ldots>$ \&first. This is a reference to the first object in the TypeArray sequence. It is saved and stored in the first member variable.

optag. A variable of type opTag. As mentioned previously, the opTag type is used as a tag into a traits class of operators. The optag variable itself serves two purposes. The first and primary purpose is to allow the compiler to deduce the data type for the optag parameter. The second purpose is to pass optional run-time information to the operators that are being applied to the TypeArray.

For example, a programmer might use for_each() to print a line of text for each item in a TypeArray. For this situation, much like we use tags to denote a TypeArray's size, we might make an optag that contains the stream to which we want to output, such as cout, and the operators would then use this parameter to produce their output.

TypeArray $<\ldots$, First+Index, .. $>()$.item. The current item in the sequence. What's actually happening is that we're instantiating the

(First+Index)'th TypeArray class in the family, then selecting the item object from that class to obtain the reference to the item being stored. 
for_each_traits $<\ldots>$ (_optag).op(...). for_each_traits is a traits object used to find the correct operator to invoke on each element of the TypeArray. The -optag is passed to the constructor of the for_each_traits object, which in turn uses it when constructing the operator functor, named $\mathrm{op}()$ here. $\mathrm{op}()$ is then executed on the current item in the TypeArray.

We won't show the implementation of for_each_traits here since it can be rather lengthy. An example of a for_each_traits class may be found in Section 8.1.

What is important to remember is that the programmer who calls for_each () must set up the for_each_traits classes so that they are fully populated with operators, one for each of the elements in the TypeArray to be iterated over. This isn't as tedious as it might sound, since it is often the case that a single for_each_traits declaration can be templatized with an integer parameter to support all of the elements in the TypeArray.

next. The next for_each_s class in the recursion to be executed. The recursion is terminated by a partial specialization of for_each_s, this time explicitly for the value of ReverseIndex $=0$.

Once again, note that the user calling for_each() doesn't need to know whether the containers are compile-time static data structures or runtime STL containers, provided that operators have been supplied for both.

This is immensely powerful because it allows users to choose STL containers when convenience and run-time behavior are more important than performance, but swap in static data structures for the same algorithms when performance is critical. Consequently, it becomes possible to incrementally add faster performance to a system without refactoring or re-architecting it.

In Draco we were able to increase the speed of our system more than 100-fold by substituting these static data structures for STL containers. We will describe in more detail how we did so in subsequent sections. Interested readers may find example uses of these algorithms in Listing 7 and Section 8.

\section{Implementation Steps, Reviewed}

Because many of the techniques demonstrated in the previous sections are fairly complex, and because the typical user will not always need to implement them all in order to solve their particular problem, we 
present in this section a brief summary of the steps typically required to use SDS with generic programming.

1 As much as possible, implement your module using generic programming with STL containers and algorithms. Stick to the algorithms that are available for static data structures. For this example we will assume that you are using for_each.

2 If you wish to use a TypeArray where every item in the array is of a different type, you will need to implement an OpTag and partial template specialization for for_each_traits. You will also need to implement a templatized operator that can operate on each of the types in your TypeArray. See Listing 12 for an example. For the simpler case where each element is of the same type, these steps are not necessary.

3 Replace your STL data structure with a TypeArray.

4 You will need to replace STL container functions such as begin() or end() with static begin() and end() functions (the former are not currently compatible with TypeArrays ${ }^{2}$ ).

5 If you wish to make a module that is simultaneously compatible with both STL and SDS containers, you will need to make sure that the third parameter to for_each can be both an operator and an OpTag. The easiest way to do this is to parameterize the class on the Iterator type, and use one implementation as the operator and the other implementation as the OpTag.

\section{Empirical Results}

As we have mentioned previously, using static data structures with generic programming allows us to incrementally add speed improvements to existing systems. The magnitude of the speed improvement varies, depending on the amount that the user is willing to determine at compile time. We have performed some tests to see what kind of performance improvements we could obtain in our own Finite Element software, Draco, for a simple steady state heat FEM problem.

At the innermost loops of the program are very small functions called basis functions, which typically come in groups and must be modular enough to be easily selectable by the user but still be extremely fast.

\footnotetext{
${ }^{2}$ This is only a matter of convenience, and we are examining ways to make iterator-access functions member functions of TypeArray to be more consistent with STL containers.
} 
A typical set of basis functions for a linear problem might look something like $f_{1}(\xi)=1-\xi$ and $f_{2}(\xi)=\xi$, and a hand-coded traditional implementation might look like,

double $f 1$ (double xsi ) \{return 1 - xsi; \}

double f2( double xsi ) \{return xsi; \}

These basis functions represent a coordinate system normalized between 0 and 1 that is more convenient to use on a local portion of the problem being modeled than traditional coordinate systems. More details about basis functions can be found in references on the Finite Element Method [14].

We compared our TMP implementation of basis functions against two traditional implementations, one using function pointers and the other using virtual functions, and obtained speed improvements of 3.5 and 11 times, respectively. This is because the traditional implementations must be stored either as function pointers or virtual functor objects in run-time arrays, whereas our TMP solution used compile time TypeArrays. Our solution has almost exactly the same performance characteristics of a solution that uses traditional inlined function calls (ie. no function pointers or virtual member functions).

The equations that use basis functions must integrate those functions over a range of values. To do so, we implemented a TMP version of the Gaussian Quadrature numerical integration algorithm. Like the basis functions, performance of this function was roughly 3.5 times and 11 times faster than the traditional implementations.

The problem with using a TMP implementation for these low-level functions is that it forces the user to either use TMP all the way at the highest levels, or to come up with some other solution to merge the higher level abstractions with the grungy TMP code. Using TMP at the highest levels would involve replacing a significant amount of perfectly respectable but non-TMP FEM code and was thus undesirable.

Our system implements a function called $\mathrm{dXsi}^{3}$ which uses the for_each() function outlined in Section 5 to iterate over the basis functions at compile time and inline nearly all of the computations for the equation, without replacing existing code. For a one-dimensional problem this layer of the code saw consistent performance improvements of 70 times the performance of the hard-coded traditional implementation. Using a profiler, we determined that the reason was because the entire equation had been inlined, resulting in nearly no function calls at all. For twodimensional problems, the performance improvement ranged from 1.8

\footnotetext{
${ }^{3} \mathrm{dXsi}$ represents $\partial \xi / \partial X$, which maps from basis function coordinates to "real" coordinates (for example, Cartesian coordinates)
} 
times to 19 times faster than the traditional implementation. The performance benefit was significantly less than that of the one-dimensional problem because we reached the compiler's inlining threshold.

This amount of inlining would not have been possible without TMP, nor would it have been feasible to integrate into our system without significantly customizing the solution for this one particular test case.

Moving higher up in our abstraction level, the element equations that use dXsi are themselves iterated over by for_each() to compute the Element matrices. Taken together, these steps constitute the vast majority of the computations that must be done to solve a Finite Element program. Overall, our one-dimensional steady state implementation performed 7 times faster than the traditional implementation that used arrays of function pointers. For the two-dimensional case, we were 171 times faster.

For those familiar with FEM, we held the following variables constant at compile time: basis functions, spatial dimensionality, size of each element, and number of degrees of freedom. Other variables were left as run-time variables, including but not limited to boundary conditions, element nodal compositions, nodal coordinates, and number of elements.

We compared the performance of our TMP solution against the previous generation of our FEM software, CMISS, written in hand-optimized Fortran. While fast, CMISS has been extended by generations of bioengineers, and as a consequence its inner loops have been corrupted with functionality specific to particular bioengineering problems. This is a direct result of the difficulty in parameterizing the Fortran algorithms for particular applications, and the performance results show that Draco is 5 times faster for the one-dimensional case and 13.5 times faster for the two-dimensional case.

The tradeoff for all of these performance gains is significantly longer compilation times. ${ }^{4}$ It's much faster to simply execute an algorithm at run-time than it is to trick the compiler into producing the same output at compile time, and so for the latter case of 171 times performance improvement, our compilation time grew from around 3.5 minutes to nearly 3 hours.

However, the standard interface shared by SDS and the STL can mitigate this effect, as it allows us to "de-optimize" certain portions of code to improve compile times for development purposes, but then optimize them later for performing fast computations.

\footnotetext{
${ }^{4}$ In addition, one might expect executable binary sizes to grow significantly due to additional inlining of code, but this was not the case for the relatively small problems that we tested with.
} 


\section{Example: An SDS-Based Event Mechanism}

Here we present an example application of SDS to the development of an event mechanism. This example was originally developed to assess the possible generality of static data structures, as events are quite different from FEM, and we choose to demonstrate it here in place of a FEM example due to the many layers of complexity in a FEM solver.

The use of an event handling mechanism as applied in the Observer pattern is a powerful method for decoupling one module from another. It allows one module writer to write a self-contained unit of execution, but still allow other code to 'hook' into the module to receive notifications when critical events occur in it. This technique is used extensively in software libraries, for example the Listener interfaces of Java 1.2 libraries.

The decoupling of the Announcer module from Listeners offers the developers of both a great deal of freedom, but it can come at a significant run-time cost. Typically, Listeners are inherited from base classes using virtual functions in order to provide the polymorphism required to allow multiple Listeners to subscribe to the same event notification. Often, the Announcers are similarly implemented, thereby ensuring at least two virtual function calls for every one event notification. This is not a problem when using coarse-grained events, but it becomes constraining for high event activity or for event handlers that are very small.

A number of implementations for event-based mechanisms exist in various programming languages, including $\mathrm{C}++$ [10]. Using GP coupled with TMP, we've designed an implementation that provides good performance when the compile-time polymorphism afforded by TMP is adequate. This brings the benefits of a well-understood traditional object-oriented tool to those who have previously not been willing to incur its run-time cost.

Our run-time cost for an event notification using this system is typically the cost of a single function call, or with a sophisticated compiler even less, as that call itself may be inlined, as shown below.

\subsection{Implementation}

In our implementation, the Announcer class has two member functions. The first is a constructor that requires a list of Listeners for instantiation. The Announcer stores this list (in the form of a beginning and ending iterator) internally.

// The announcer

template <class EventType, class TypeArrayIt1, class TypeArrayIt2> class Announcer \{ 
typedef typename TypeArrayIt1::ArrayTag ArrayTag;

TypeArrayIt1_begin;

TypeArrayIt2 _end;

public:

// Constructor

inline Announcer( TypeArrayIt1 begin, TypeArrayIt2 end )

: _begin(begin), _end(end) \{\}

void announce( EventType e ) \{

Announcer_optag<EventType, ArrayTag> optag(e);

\}

for_each (_begin, _end, optag );

\};

Listing 10: Announcer implementation

The second function is the actual announce() mechanism. When called, it uses the for_each() algorithm we introduced previously to iterate over all of the Listeners and invoke the Announcer_operator on each, which simply notifies them of the event by passing the event to their accept() member functions. The Announcer_operator will be demonstrated in the next source code listing (Listing 11).

The Announcer_optag is used to refer to a sequence of operators that are stored in the for_each_traits family of classes used by for_each. These operators are the functions triggered by the announcer in each listener. For each item in the TypeArray of Listeners, for_each() will look up the operator in the for_each_traits that has the same index number and apply it to the item. Setting up the for_each_traits family looks like Figure 11.

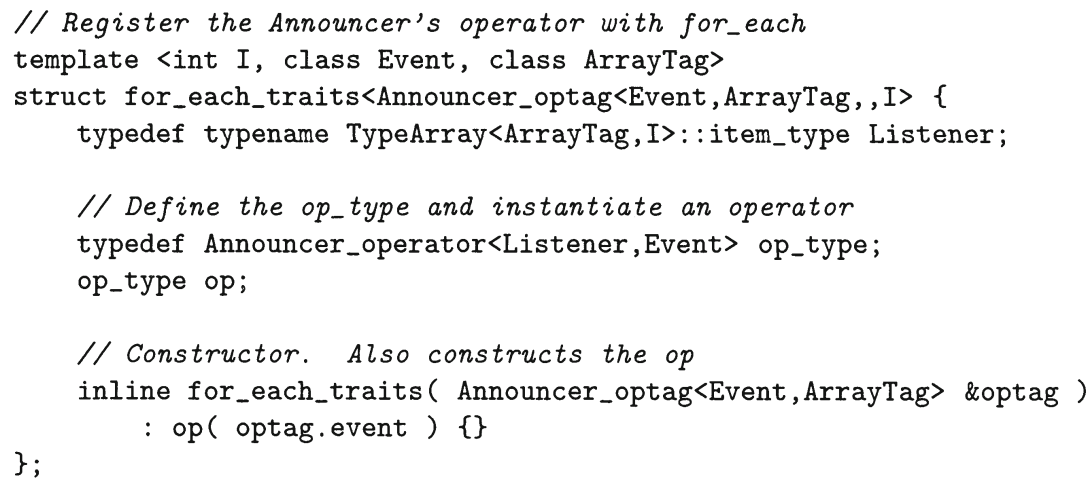

Listing 11: for_each_traits for Announcer 
Notice that op_type in for_each_traits is our Announcer_operator class. This class will be invoked for every element in the TypeArray. The implementation of the Announcer_operator is very simple and looks like this:

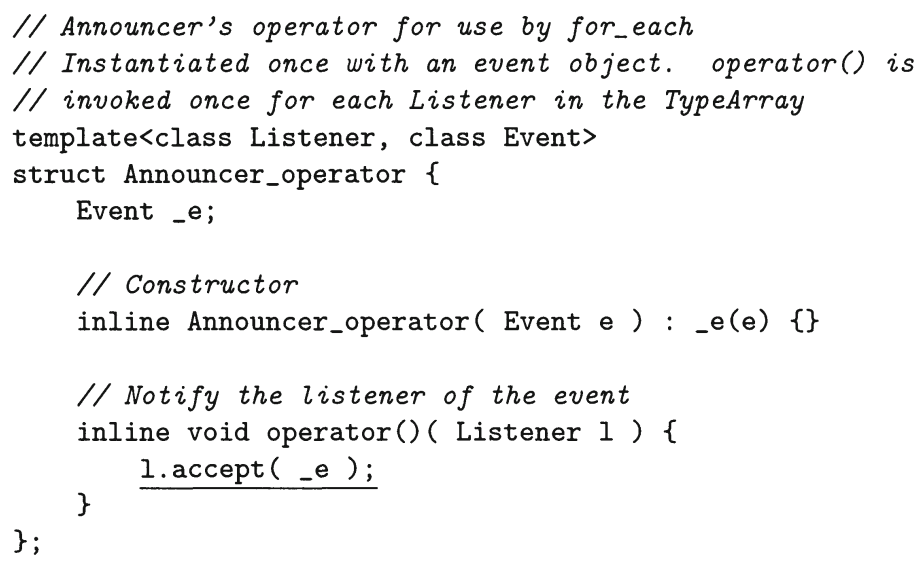

Listing 12: Templatized operator called by for_each() to activate listeners

\subsection{Usage}

Here is a complete example program to demonstrate the usage of this technique.

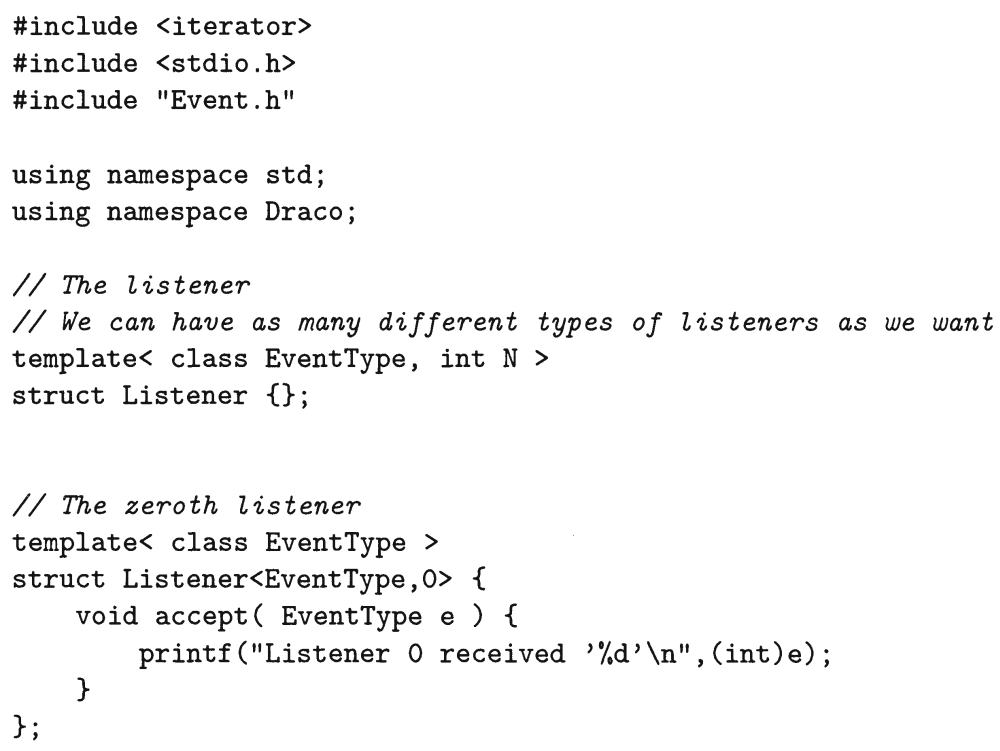




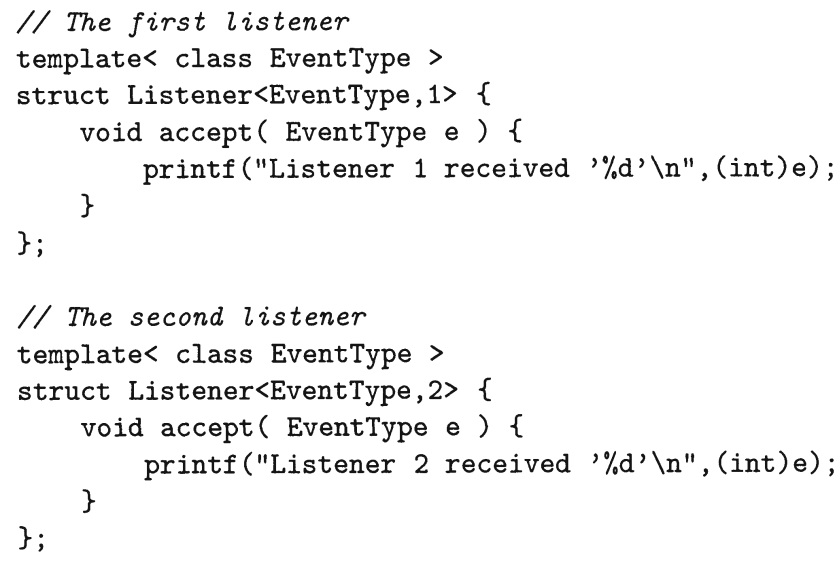




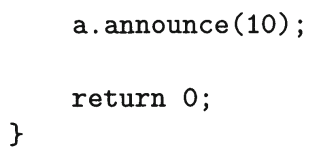

The output to the above program will be

Listener 0 received: '10'

Listener 1 received: '10'

Listener 2 received: '10'

\subsection{Performance}

Here is the output of the KCC compiler for the above program [9]. This code was obtained by using the --keep_gen_c option in KCC to retain the intermediate $\mathrm{C}$-code used in compiling the $\mathrm{C}++$ code, then running that code through a name de-mangler and cleaning the output manually. Important bits are underlined.

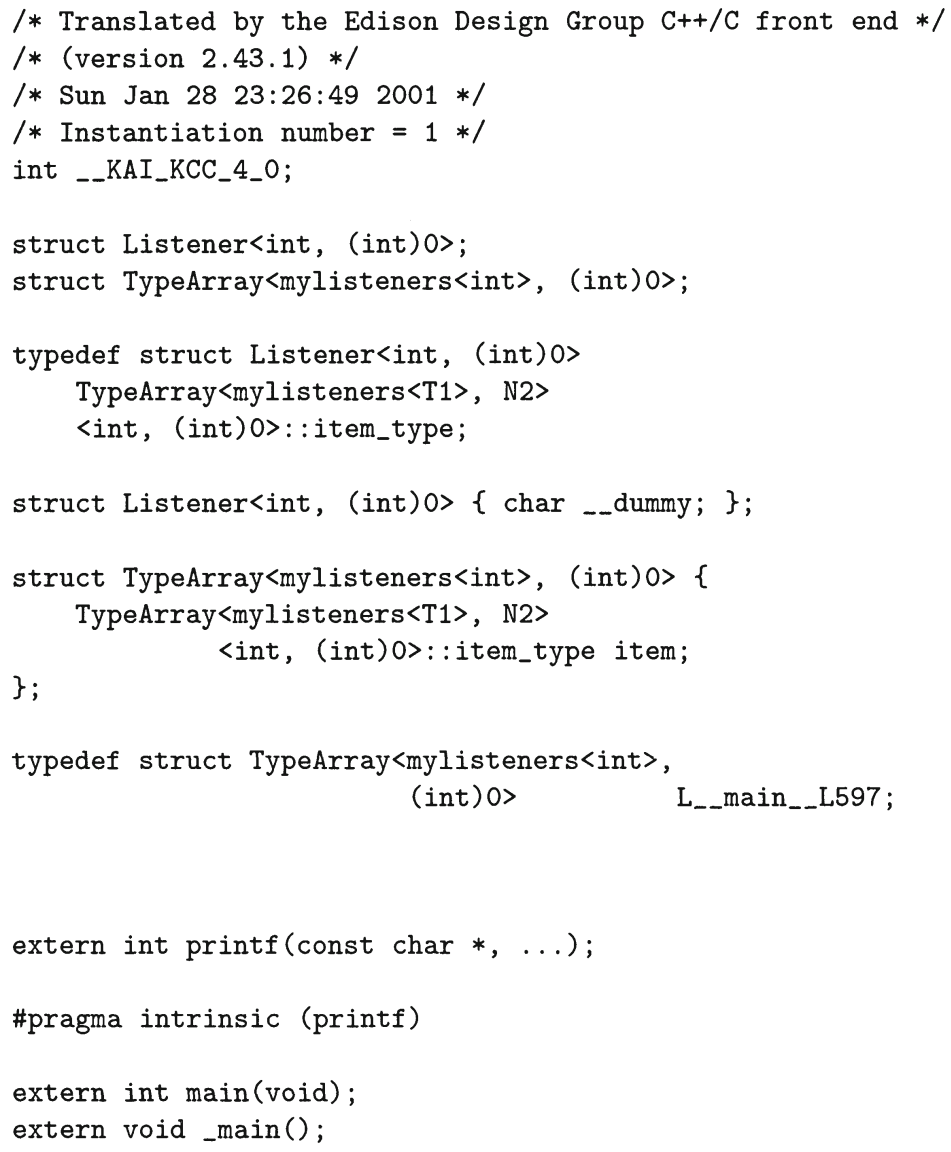




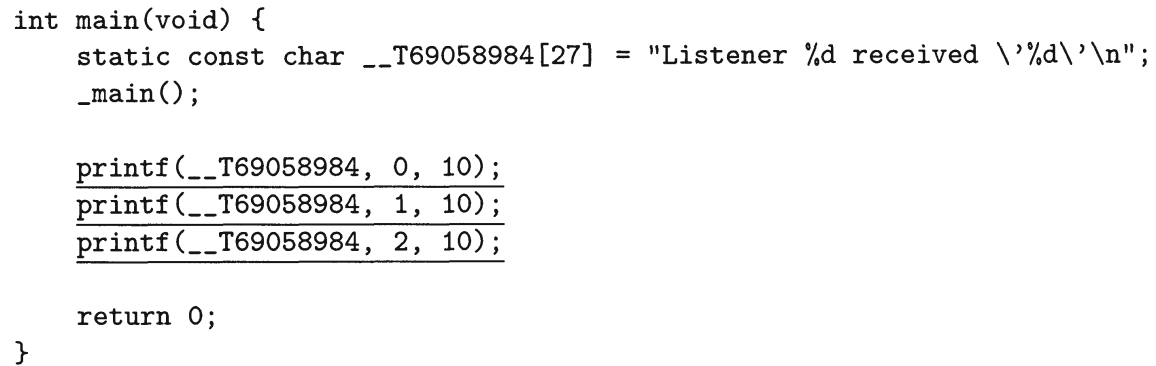

Listing 13: Intermediate C output for Event

As you can see, the entire Event-handling mechanism has been optimized away, leaving in its place only the three calls to printf(). The rest of the code in the output is mostly just structure definitions and will be stripped away by the $\mathrm{C}$ compiler when it generates the machine language output. This means that the entire cost of the software decomposition has been eliminated by using Generic Programming with static data structures.

\section{Discussion}

Static data structures are not applicable to every case that one might wish to use them. They're particularly useful if each item in a data structure must be of a different type, or if manual control of loop unrolling is required. However, the following conditions must be met before replacing an STL data structure with a static data structure:

1 The size of the container is known at compile time.

2 Explicit loop unrolling is desired.

3 Non-mutating algorithms that have a return type determinable at compile-time are required. For example, any algorithm that returns an iterator is disqualified by this rule, since the type of the iterator is a function of its position in the container at run-time. ${ }^{5}$

These restrictions suit our application well since, like many scientific computation applications, the computational layers of FEM unfold like an onion and consist of many nested loops with many elements that can

\footnotetext{
${ }^{5}$ It is possible to bypass this restriction if the types contained in the TypeArray are strictly integer types since $\mathrm{C}++$ allows the compiler to perform comparisons of integer template parameters, but this base case is probably of limited utility in real world applications.
} 
be evaluated at compile time. We can leverage the same SDS technique for several layers of the system moving outward since we know the loop boundaries of these at compile time, as the discretization of the problem results in predefined loop bounds.

In the future, we'd like to expand our repertoire of static data structures and compatible algorithms. We have thoroughly examined only one static data structure in our system, so we haven't explored the genericity of our GP static data structure algorithms yet. We've investigated a static data structure that looks like a linked list, similar to heterogenous type lists [4], but we have not determined if it has properties that make it better suited to other problems than the TypeArray. We have primarily focused on making TypeArrays interchangeable with standard STL algorithms.

Another constraint of our design is that the algorithms are more specific to their applications than are their STL counterparts. For example, the current implementation of for_each() will only work with TypeArray, but not with any other static data structures. This is because the language of Template Metaprogramming is much more restrictive than procedural programming languages.

Although additional research in this area may be able to overcome this limitation, it has not been limiting for our purposes. The primary benefit of emulating STL algorithms is that we can easily interchange dynamic data structures and static data structures to manage performance, without having to change our system's modular decomposition.

\section{Conclusion}

We needed a software architecture that would enable us to build a highly flexible Finite Element system in a straightforward way, focusing first on a clean design and correct functionality and letting us postpone decisions about performance until later. Our ideal was to develop freely with the STL, and then with some additional effort slip in a TMP solution later to enhance performance.

Existing solutions tend to be implemented with TMP, or with generic programming techniques, but not with both. Our solution unifies the two and can provide performance that can be incrementally added to a system at relatively little cost.

Our solution consists of two parts. The first, called Static Data Structures, is a new type of container that can hold references to objects of more than one type. This trait enables the container to hold references to various partial specializations of the same base templatized class, all 
of which are conceptually related but treated as distinct types by the container.

The second part of the solution is to provide algorithms that can operate on these data structures in a way that is compatible with the algorithms of the STL. These algorithms have the same behavior, name, and number of arguments as their STL brethren, but maintain a distinct type signature usable only by static data structures. This enables the user to cleanly augment existing STL-based code with higher performance TMP objects, without rewriting existing code.

We have validated the performance of our implementation in our FEM package, Draco. We have achieved performance improvements ranging between 7 and 171 times faster than similar implementations written modularly in C. Performance was between 5 and 13.5 times faster than legacy hand-optimized Fortran code. In neither case was our code less modular than the code we compared against.

\section{Acknowledgements}

The authors wish to thank the reviewers, Scott Baden, and Emily Winch for their pointers and expert comments on drafts of this paper. We would also like to thank Aleksey Gurtovoy for sending us a preview of his unpublished work on the MPL.

\section{Appendix: TypeArray}

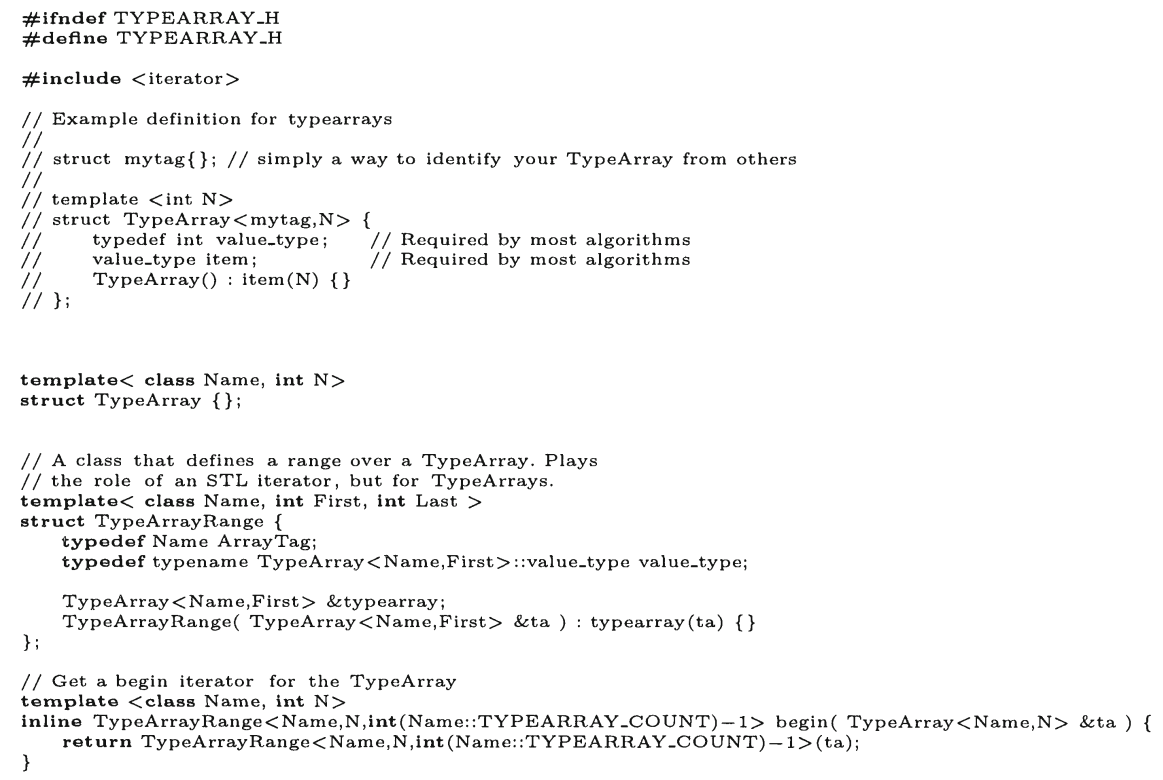




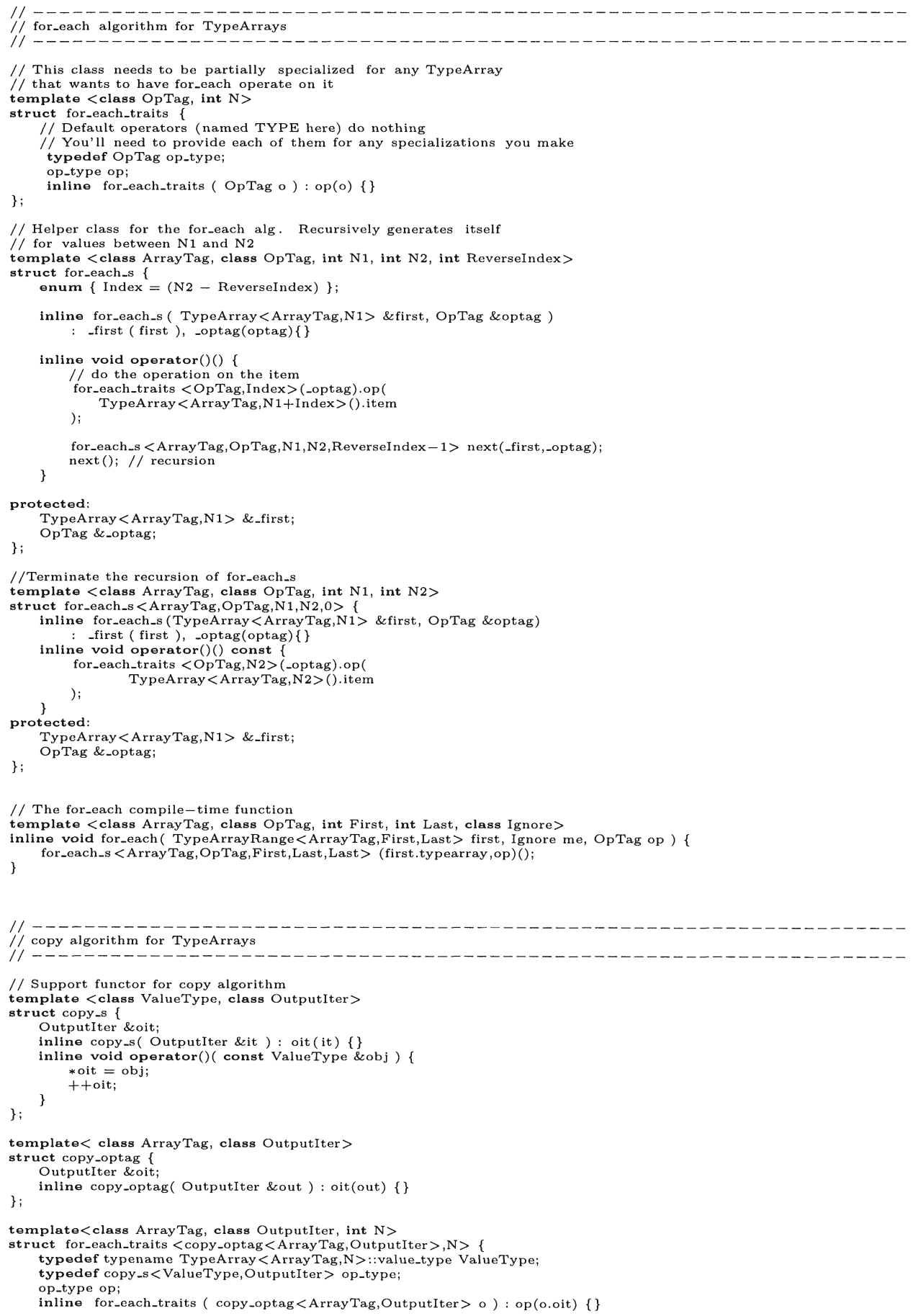




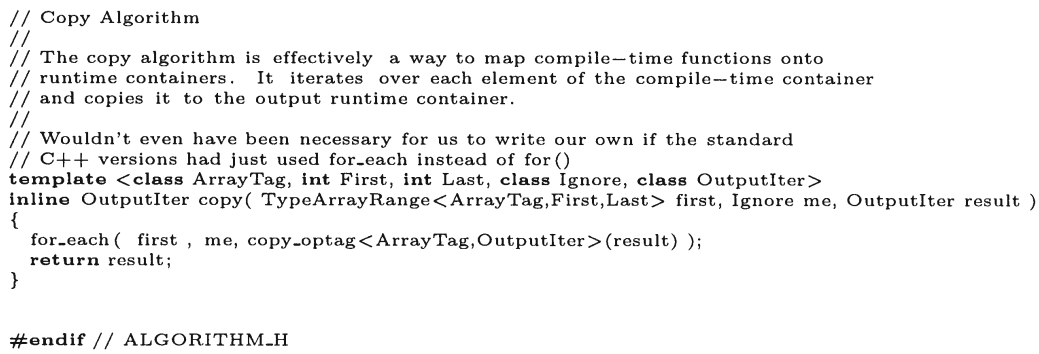

\section{References}

[1] Boost. http://www.boost.org, 2002.

[2] Michael C. Burton. Exploring extensibility and performance in a generic programming finite element system. Masters Thesis, University of California, San Diego, Department of Computer Science and Engineering, June 2001, June 2001.

[3] Kryzstof Czarnecki and Ulrich W. Eisenecker. Metalisp. http://home.tonline.de/home/Ulrich.Eisenecker/meta.htm, 1998.

[4] Krzysztof Czarnecki and Ulrich W. Eisenecker. Generative Programming: Methods, Tools, and Applications. Addison-Wesley Publishing Co., Reading, Mass., 2001.

[5] Ulrich W. Eisenecker, Frank Blinn, and Krzysztof Czarnecki. A solution to the constructor-problem of mixin-based programming in C++. In First Workshop on C++ Template Programming, Erfurt, Germany, 102000.

[6] Aleksey Gurtovoy and David Abraham. The Boost C++ Metaprogramming Library. http://www.mywikinet.com/mpl/, 32002.

[7] Jaakko Jarvi. Tuples and multiple return values in C++. Technical report, No. 249, TUCS, March 1999. http://www.tucs.fi/publications/techreports/TR249.php.

[8] Neil D. Jones, Carsten K. Gomard, and Peter Sestoft. Partial Evaluation and Automatic Program Generation. Prentice Hall International, International Series in Computer Science, June 1993. ISBN number 0-13-020249-5 (pbk).

[9] Kuck and Associates. Kuck and Associates C++ User's Guide. 2000.

[10] David Notkin, David Garlan, William G. Griswold, and Kevin Sullivan. Adding Implicit Invocation to Languages: Three Approaches. In Object Technologies for Advanced Software, volume 742 of Lecture Notes in Computer Science, pages 489-510. First JSSST International Symposium, November 1993.

[11] T. L. Veldhuizen. C++ templates as partial evaluation. In ACM SIGPLAN Workshop on Partial Evaluation and Semantics-Based Program Manipulation, 2000.

[12] Todd L. Veldhuizen. Expression templates. C++ Report, 7(5):26-31, June 1995. Reprinted in C++ Gems, ed. Stanley Lippman.

[13] Emily Winch. Heterogenous lists of named objects. In Second Workshop on C++ Template Programming, Tampa, Florida, 102001.

[14] O. C. Zienkiewicz and R. Taylor. The finite element method. McGraw-Hill, 1989. 